\title{
DOAK-Empfehlungen bei Thrombose
}

In der aktualisierten Leitlinie zur Diagnostik und Therapie von venösen Thromboembolien stehen direkte orale Antikoagulanzien (DOAK) jetzt gleichberechtigt neben den Vitamin-K-Antagonisten.

Jeder klinische Verdacht auf eine Venenthrombose muss umgehend abgeklärt werden. Die Leitlinie gibt dafür im Wesentlichen zwei Wege vor: Am Anfang steht immer die Abschätzung der klinischen Wahrscheinlichkeit mit einem standardisierten Score wie dem Wells-Score. Werden im Wells-Score weniger als zwei Punkte erreicht, besteht ein geringes

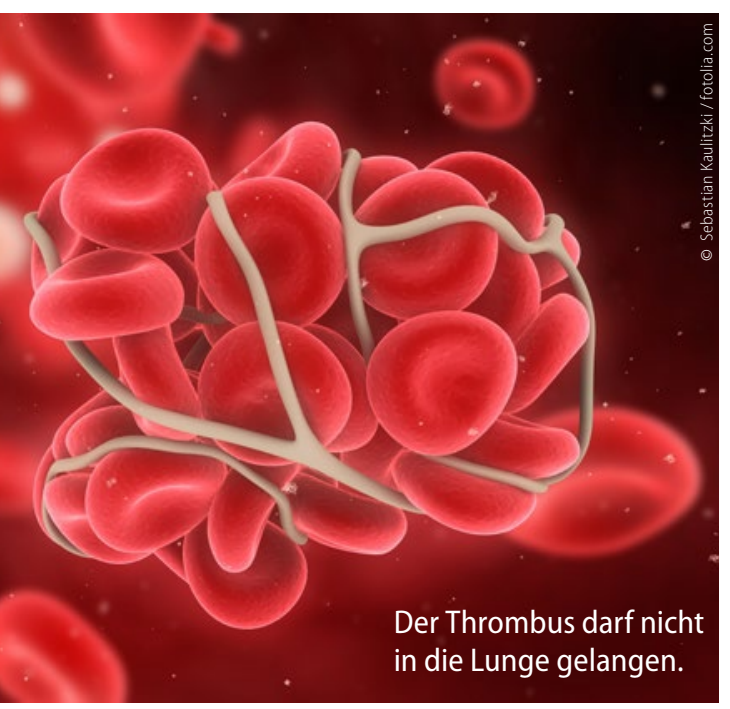

Thromboserisiko, ab zwei Punkten liegt das Risiko bei mindestens 30\%.

Von der Punktzahl hängt auch das weitere Vorgehen $a b$. Wenn sie unter zwei liegt, sollte ein D-Dimer-Test gemacht werden. Fällt der Test negativ aus, ist keine weitere Thrombosediagnostik erforderlich. Ist er positiv, schließt sich eine bildgebende Untersuchung an, in der Regel ein Kompressionsultraschall (KUS).

Patienten mit zwei oder mehr Punkten im Wells-Score müssen dagegen sofort eine bildgebende Untersuchung erhalten. „Auf das Ergebnis des D-Dimer-Tests zu warten, kostet Zeit - und diese Patienten eventuell das Leben“, warnte Prof. Dr. Edelgard Lindhoff-Last, Frankfurt. Besteht ein klinisch begründeter Verdacht, und ist nicht sofort ein KUS verfügbar, empfiehlt die Expertin, dem Patienten zur Überbrückung noch in der Praxis ein niedermolekulares Heparin (NMH) zu geben. Die Sonografie muss aber auch in diesem Fall innerhalb von 24 Stunden erfolgen.

\section{Therapeutische Antikoagulation}

Steht die Diagnose einer tiefen Venenthrombose (TVT), muss sofort mit der therapeutischen Antikoagulation begonnen werden, um ein weiteres Wachstum des Thrombus und vor allem eine Einschwemmung in die Lungenstrombahn zu verhindern. In der überarbeiteten Leitlinie sind dafür erstmals die vier direkten oralen Antikoagulanzien (DOAK) Dabigatran, Rivaroxaban, Apixaban und Edoxaban genannt, und zwar gleichwertig mit den Vitamin-K-Antagonisten (VKA). Zu den Vorteilen der DOAK gehören die fehlende Notwendigkeit von Gerinnungskon- trollen sowie kürzere Halbwertszeiten. Zur Initialtherapie können wie gewohnt $\mathrm{NMH}$, Fondaparinux oder - bei schwerer Niereninsuffizienz - unfraktioniertes Heparin (UFH) eingesetzt werden, ggf. überlappend mit einem VKA. Alternativ können die DOAK Rivaroxaban und Apixaban gegeben werden, und zwar von Anfang an als Monotherapie. Nach drei Wochen bzw. nach einer Woche muss die Dosis allerdings auf die Erhaltungsdosis reduziert werden. Bei Verwendung von Dabigatran oder Edoxaban muss zunächst fünf Tage lang mit einem NMH, UFH oder Fondaparinux allein behandelt und dann auf das DOAK umgestellt werden.

\section{Erhaltungstherapie}

Die Erhaltungstherapie nach einer ersten Thrombose sollte über drei bis sechs Monate erfolgen. Bei Patienten mit einem klar identifizierbaren und passageren Triggerfaktor wie einer OP wird die Antikoagulation i.d.R. nach dieser Zeit beendet.

Unter bestimmten Umständen kann eine verlängerte Erhaltungstherapie in Betracht gezogen werden. Bei fortbestehendem Triggerfaktor (z.B. aktive Tumorerkrankung) wird das Rezidivrisiko als so hoch angesehen, dass eine verlängerte Erhaltungstherapie in der Regel indiziert ist, sofern nicht ein sehr hohes Blutungsrisiko dagegen spricht. Weitere Kriterien für eine Fortführung der Therapie sind unklare Genese, proximale Thrombuslokalisation und die Patientenpräferenz. DOAK können auch zur verlängerten Erhaltungstherapie eingesetzt werden. (Beate Schumacher)

Praxis Update Allgemeinmedizin, München, April 2016

\section{DATEN UND FAKTEN}

\subsection{3}

Menschen in Deutschland starben im Jahr 2014 nach Angaben des Statistischen Bundesamts in Wiesbaden an einem nicht näher bezeichneten Schlaganfall (ICD-10-Diagnose 164). Der Schlaganfall belegt damit Platz 10 der häufigsten Todesursachen. Die Zahl der Schlaganfalltoten ist seit Jahren rückläufig: 1998 fielen noch knapp 52000 Menschen einem zerebrovaskulären Ereignis zum Opfer. Ein Schlaganfall war damals noch die vierthäufigste Todesursache.

\section{INFOS IM INTERNET}

Alle Folgen der Sommer-Akademie finden Sie im Web unter www.aerztezeitung.de Das aktuelle Update „Rekanalisierende Therapie” zur S1-Leitlinie "Akuttherapie des ischämischen Schlaganfalls" gibt es bei der DGN unter: www.dgn.org/leitlinien/aktualisierungen

Viele Informationen für Patienten bietet die Stiftung Deutsche Schlaganfall-Hilfe auf: www.schlaganfall-hilfe.de

Ärzte dürfte die Seite der Deutschen Schlaganfall-Gesellschaft (www.dsg-info.de) und die des Kompetenznetzes Schlaganfall interessieren:

www.kompetenznetz-schlaganfall.de 